\title{
Experimental Characterization of Unsharp Qubit Observables and Sequential Measurement Incompatibility via Quantum Random Access Codes
}

\author{
Hammad Anwer $\odot,{ }^{1}$ Sadiq Muhammad $\odot,{ }^{1}$ Walid Cherifi, ${ }^{1}$ Nikolai Miklin $\odot,{ }^{2}$ \\ Armin Tavakoliø, ${ }^{3}$ and Mohamed Bourennane ${ }^{1}$ \\ ${ }^{1}$ Department of Physics, Stockholm University, S-10691 Stockholm, Sweden \\ ${ }^{2}$ Institute of Theoretical Physics and Astrophysics, National Quantum Information Center, \\ Faculty of Mathematics, Physics and Informatics, University of Gdansk, 80-952 Gdánsk, Poland \\ ${ }^{3}$ Département de Physique Appliquée, Université de Genève, CH-1211 Genève, Switzerland
}

(Received 17 January 2020; revised 30 April 2020; accepted 23 July 2020; published 19 August 2020)

\begin{abstract}
Unsharp measurements are increasingly important for foundational insights in quantum theory and quantum information applications. Here, we report an experimental implementation of unsharp qubit measurements in a sequential communication protocol, based on a quantum random access code. The protocol involves three parties; the first party prepares a qubit system, the second party performs operations that return both a classical and quantum outcome, and the latter is measured by the third party. We demonstrate a nearly optimal sequential quantum random access code that outperforms both the best possible classical protocol and any quantum protocol that utilizes only projective measurements. Furthermore, while only assuming that the involved devices operate on qubits and that detected events constitute a fair sample, we demonstrate the noise-robust characterization of unsharp measurements based on the sequential quantum random access code. We apply this characterization towards quantifying the degree of incompatibility of two sequential pairs of quantum measurements.
\end{abstract}

DOI: 10.1103/PhysRevLett.125.080403

Introduction.-Textbook measurements in quantum theory are represented by complete sets of orthogonal projectors. However, general measurements in quantum theory are described by positive operator-valued measures (POVMs), i.e., an ordered set of positive operators $\left\{M_{i}\right\}_{i}$ with normalization $\sum_{i} M_{i}=\mathbb{1}$. Evidently, projective measurements are instances of POVMs but not all POVMs are projective measurements. These nonprojective measurements are well defined in Hilbert spaces of fixed dimension (otherwise they can be viewed as projective measurements in a larger space [1]). They are foundationally interesting and relevant to many phenomena and applications of quantum theory.

Some nonprojective measurements are extremal in the space of all POVMs with fixed Hilbert space dimension and number of outcomes i.e., they cannot be simulated with stochastic implementation of other measurements [2]. Whereas such POVMs have been studied in broad contexts [2-11], far from all nonprojective measurements are of this type. In fact, many interesting POVMs are unsharp measurements, in the sense that they are weaker (noisy)

Published by the American Physical Society under the terms of the Creative Commons Attribution 4.0 International license. Further distribution of this work must maintain attribution to the author(s) and the published article's title, journal citation, and DOI. Funded by Bibsam. variants of projective measurements. By suitably tuning the noise parameter (sharpness), an experimenter can control the information-disturbance trade-off [12]; continuously from extracting no information and inducing no disturbance (noninteractive measurement) to extracting maximal information and inducing maximal disturbance (sharp projective measurement). Sequential unsharp measurements that individually induce only a small disturbance can be used for real-time monitoring of the evolution of single quantum systems [13-16]. When sufficiently frequent, such sequences effectively constitute continuous measurements, which have broad relevance in quantum information science (see, e.g., the review in Ref. [17]). Two key application of sequential unsharp measurements are adaptive measurement protocols [18,19] and quantum feedback protocols [20-22]. Interestingly, such sequences are also versatile as they can be used to realize the most general quantum measurements [23]. Moreover, unsharp measurements have prominent roles in a number of other topics including weak values [24], entanglement amplification [25], quantum random number generation [26], tests of the memory capacity of classical systems [27] and sequential quantum correlations [12,28-31]. This has prompted a number of experiments focused on the implementation of incompatible measurements [32-34], quantum contextuality [30], and quantum nonlocality [35-37].

Recently, Refs. [38,39] considered unsharp measurements in a sequential implementation of a frequently 
encountered communication task known as a quantum random access code (QRAC) [40-42]. In a (Q)RAC, a sender, Alice, receives two input bits $\left(x_{0}, x_{1}\right)$ which she encodes into a (qu)bit that is sent to a receiver, Bob. Bob receives an input bit $y$ and then attempts to choose his output $b$ such that it equals to Alice's $y$ th bit, i.e., $b=x_{y}$. In an optimal classical protocol, Alice always sends $x_{0}$; thus Bob succeeds when $y=0$ and takes a random guess when $y=1$, leading to an average success probability of 0.75 . However, a quantum advantage is obtained if Alice prepares four qubit states forming a square on the equator of the Bloch sphere and Bob measures two suitably aligned Pauli observables, resulting in a success rate of $\approx 0.85$. From an alternative perspective, a QRAC can be viewed as a certification tool that allows an experimenter to characterize the involved preparation and measurement devices solely from its success rate, while assuming only that the setup operates on qubits [43].

However, unsharp measurements in standard QRACs are unremarkable as their outcome statistics can be simulated by a measurement device that stochastically implements projective measurements. Therefore, Refs. [38,39] considered a sequential scenario (see Fig. 1) in which the postmeasurement state of Bob is relayed to another receiver, Charlie, who receives an input bit $z$ and analogously attempts to recover the $z$ th bit of Alice. Thus, Alice sequentially implements a QRAC with Bob and Charlie in the respective order. Here, unsharp measurements become indispensable: in order for both QRACs to achieve a high success rate, Bob must interact with the incoming system in such a way that sufficient information is extracted to power his guess of $x_{y}$, while simultaneously the disturbance is limited to allow Charlie to accurately guess $x_{z}$. Furthermore, it was shown [38,39] that sequential QRACs can serve as certification tools for characterizing the unsharpness of Bob's operations while only assuming that the states are qubits.

In this Letter, we report experimental implementation of sequential QRACs using measurements of tunable unsharpness and demonstrate nearly optimal quantum correlations that outperform both all classical protocols as well as all quantum protocols based only on projective qubit measurements. We harvest these quantum communication

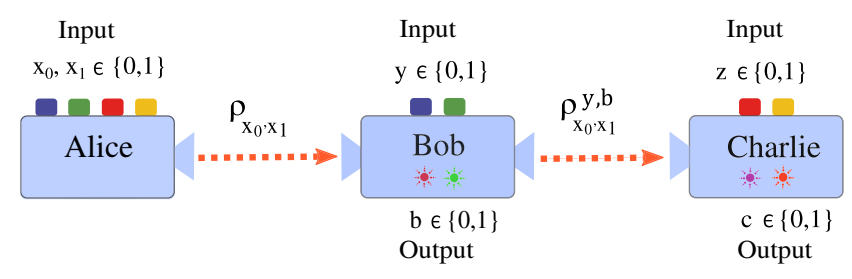

FIG. 1. Alice receives two bits $x_{0}, x_{1}$ and sends the qubit state $\rho_{x_{0}, x_{1}}$ to Bob who receives an input $y$ and produces a classical output $b$ and a quantum output $\rho_{x_{0}, x_{1}}^{y, b}$, which is measured by Charlie according to his input $z$, yielding an outcome $c$. advantages to certify the unsharpness parameter by confining it to a narrow interval. Subsequently, we theoretically develop and experimentally demonstrate how the sequential QRACs can be applied to quantify the degree of incompatibility [44] between two sequential pairs of quantum measurements.

Scenario and theoretical background.-Based on Refs. [38,39], we describe the sequential QRAC experiment. It involves three parties, Alice, Bob, and Charlie (see Fig. 1). Alice receives an input $x \equiv x_{0}, x_{1} \in\{0,1\}$ and prepares some uncharacterized qubit state denoted $\rho_{x}$, which she sends to Bob. Bob receives an input $y \in$ $\{0,1\}$ and performs a corresponding operation on $\rho_{x}$. This operation produces a classical output $b \in\{0,1\}$ and some postoperation qubit state denoted $\rho_{x}^{y, b}$, which is sent to Charlie. Charlie receives an input $z \in\{0,1\}$ and then measures $\rho_{x}^{y, b}$, yielding an outcome $c \in\{0,1\}$. All inputs $(x, y, z)$ are statistically independent and uniformly distributed. The limit of many rounds yields conditional probability distributions $p(b, c \mid x, y, z)$.

The conditional probability distributions $p(b, c \mid x, y, z)$ are used to evaluate the success rate of two QRACs: one between Alice and Bob, and one between Alice and Charlie. The former is successful when $b=x_{y}$ and the latter is successful when $c=x_{z}$. The two respective success rates read

$$
\begin{aligned}
W_{\mathrm{AB}} & =\frac{1}{8} \sum_{x, y} P\left(b=x_{y} \mid x, y\right), \\
W_{\mathrm{AC}} & =\frac{1}{8} \sum_{x, z} P\left(c=x_{z} \mid x, z\right) .
\end{aligned}
$$

Note that we can always take $W_{\mathrm{AB}}, W_{\mathrm{AC}} \in\left[\frac{1}{2}, 1\right]$. Evidently, $W_{\mathrm{AB}}$ is independent of Charlie. However, $W_{\mathrm{AC}}$ is not independent of Bob because he operates on the system before it reaches Charlie.

Bob's two operations $(y=0,1)$ are described by the notion of a quantum instrument [45], which captures both the measurement statistics and the evolution of the measured state. A quantum instrument is defined as an ordered set of trace-non-increasing completely positive maps $\left\{\Lambda_{b \mid y}\right\}_{b}$ with the property that for any state $\rho$ it holds that $p(b \mid y)=\operatorname{tr}\left[\Lambda_{b \mid y}(\rho)\right]$. Having observed the classical output $b$, the quantum output of the instrument is $\rho^{y, b}=\Lambda_{b \mid y}(\rho) / \operatorname{tr}\left[\Lambda_{b \mid y}(\rho)\right]$. Since we consider qubits and Bob has binary outcomes, the extremal quantum instruments are written as $\Lambda_{b \mid y}(\rho)=K_{b \mid y} \rho K_{b \mid y}^{\dagger}$, where $\left\{K_{b \mid y}\right\}_{b}$ are Kraus operators satisfying $\sum_{b} K_{b \mid y}{ }^{\dagger} K_{b \mid y}=\mathbb{1}$, with the convenient property that $K_{b \mid y}{ }^{\dagger} K_{b \mid y}=B_{b \mid y}$ where $\left\{B_{b \mid y}\right\}_{b}$ are the two POVMs of Bob [46]. For simplicity, we can represent Bob's measurements in terms of two observables which, in general, read $B_{y} \equiv B_{0 \mid y}-B_{1 \mid y}=\alpha_{y} \mathbb{1}+\vec{n}_{y} \cdot \vec{\sigma}$, where $\vec{n}_{y}$ are Bloch vectors, $\vec{\sigma}$ are the Pauli matrices, and $\left|\alpha_{y}\right| \leq 1-\left|\vec{n}_{y}\right|$. The sharpness of Bob's measurements is 
defined as $\eta_{y}=\left|\vec{n}_{y}\right|$. Notice that for $\eta_{y} \in\{0,1\}$, the measurements are noninteractive and sharp, respectively, whereas $\eta_{y} \in(0,1)$ corresponds to intermediate cases. We consider the case of $\eta \equiv \eta_{0}=\eta_{1}$. We emphasize that one can stochastically simulate Bob's unsharp POVMs using only projective measurements, but one cannot simulate his quantum instrument in this manner. Therefore, we can distinguish a projective simulation from a genuine unsharp measurement by considering both the classical and quantum output.

By inspecting the witnesses $\left(W_{\mathrm{AB}}, W_{\mathrm{AC}}\right)$, one may characterize the sharpness parameter $\eta$. References $[38,39]$ showed that for a given value of $W_{\mathrm{AB}}$, the optimal value of $W_{\mathrm{AC}}$ in quantum theory is given by

$$
W_{\mathrm{AC}}=\frac{1}{8}\left(4+\sqrt{2}+\sqrt{16 W_{\mathrm{AB}}-16 W_{\mathrm{AB}}^{2}-2}\right),
$$

and that such an optimal pair implies a precise value of $\eta$. However, in the experimentally realistic case in which perfectly optimal quantum correlations are not relevant, a suboptimal witness pair can be used to deduce upper and lower bounds on $\eta$,

$$
\begin{array}{r}
\eta \geq \sqrt{2}\left(2 W_{\mathrm{AB}}-1\right) \\
\eta \eta_{\min }, \\
\eta \leq 2 \sqrt{\left(2+\sqrt{2}-4 W_{\mathrm{AC}}\right)\left(2 W_{\mathrm{AC}}-1\right)} \equiv \eta_{\max } .
\end{array}
$$

Thus, the closer the experimentally observed correlations are to the optimal ones in Eq. (2), the narrower is the interval $I\left(W_{\mathrm{AB}}, W_{\mathrm{AC}}\right) \equiv\left[\eta_{\min }, \eta_{\max }\right]$ to which we can confine the sharpness $\eta$.

Experiment.-The optimal quantum correlations (2) are obtained with a unique quantum strategy (up to a global unitary) [38]. Alice needs to prepare four states forming a square on a great circle on the Bloch sphere. For simplicity we choose the $x z$ plane and Alice's four states $\left|\psi_{x_{0} x_{1}}\right\rangle=\cos \alpha_{x_{0} x_{1}}|0\rangle+\sin \alpha_{x_{0} x_{1}}|1\rangle$, corresponding to the four values $\{(\pi / 8),-(3 \pi / 8),(9 \pi / 8),(5 \pi / 8)\}$ of $\alpha_{x_{0} x_{1}}$, respectively, where $\rho_{x}=\left|\psi_{x_{0} x_{1}}\right\rangle\left\langle\psi_{x_{0} x_{1}}\right|$. Similarly, the optimal quantum instruments of Bob correspond to the Kraus operators $K_{b \mid y}=\sqrt{\left(\mathbb{1}+(-1)^{b} B_{y}\right) / 2}$ for a suitably chosen $\eta$, where $B_{y} \in\left\{\eta \sigma_{x}, \eta \sigma_{z}\right\}$ are the corresponding observables of Bob. The quantum output is sent to Charlie whose observables are two complementary projective measurements $C_{0}=\sigma_{x}$ and $C_{1}=\sigma_{z}$. In an ideal experiment, for every $\eta$, we obtain the witness pair,

$W_{\mathrm{AB}}=\frac{2+\sqrt{2} \eta}{4}, \quad W_{\mathrm{AC}}=\frac{4+\sqrt{2}+\sqrt{2-2 \eta^{2}}}{8}$,

which satisfies the optimality condition (2).

We implemented this optimal strategy, using singlephoton polarization qubits where the computational basis corresponds to horizontal $(H)$ and vertical $(V)$ polarization, i.e., $|H\rangle \equiv|0\rangle$ and $|V\rangle \equiv|1\rangle$. The complete optical setup is

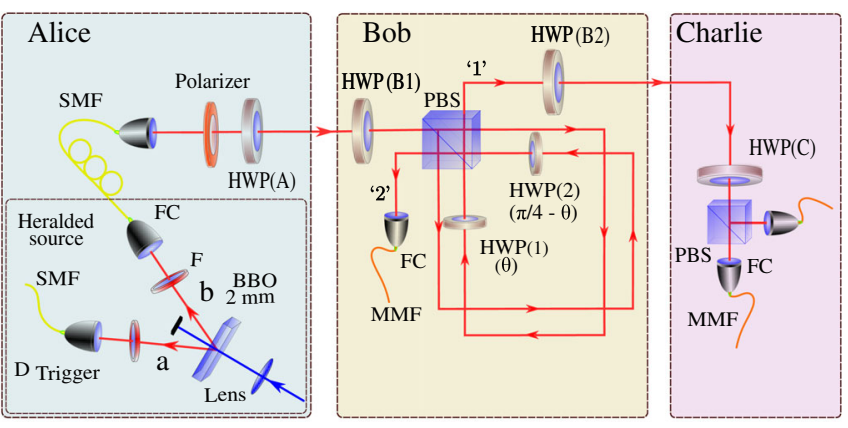

FIG. 2. Experimental setup. Alice prepares her states using a heralded photon source, a polarizer and a half-wave plate HWP (A). Bob's instrument is realized by a shifted Sagnac interferometer where the sharpness parameter $\eta=\cos (4 \theta)$ is tuned using half-wave plates $\operatorname{HWP}(1)$ and $\operatorname{HWP}(2)$. $\mathrm{HWP}(\mathrm{B} 1)$ and $\mathrm{HWP}(\mathrm{B} 2)$ are used to switch between the observables $B_{0}$ and $B_{1}$ as well as selecting the output corresponding to the outcome $b=0$ and $b=1$. Charlie performs projective (sharp) measurements on the received qubit from Bob using a $\operatorname{HWP}(C)$ and a polarization beam splitter (PBS).

shown in Fig. 2. Alice's preparation device also encloses a heralded single photon source that produces photons at wavelength $780 \mathrm{~nm}$ through spontaneous parametric down conversion (SPDC) by pumping a type-I beta barium borate (BBO) single crystal of thickness $2 \mathrm{~mm}$ using $390 \mathrm{~nm}$ fs laser pulses. Time correlated idler and signal photons are spectrally and spatially purified by passing through $3 \mathrm{~nm}$ (FWHM) wide optical filters $(F)$ and coupling into single mode fibers (SMF), respectively. The idler photons in mode " $a$ " are detected by an avalanche photodiode (APD), marked as $D_{\text {Trigger }}$, with detection efficiency $\sim 60 \%$, which produces a trigger signal indicating the presence of a photon in mode " $b$." Alice prepares this photon in one of the four desired states $\left|\psi_{x_{0}, x_{1}}\right\rangle$ using a polarizer when it

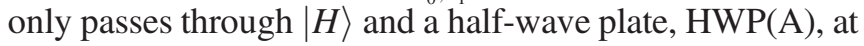
angles $11.25^{\circ},-11.25^{\circ} 33.75^{\circ}$ and $-33.75^{\circ}$, respectively, and sends it to Bob.

Bob's unsharp measurements on the received photons are performed using a shifted Sagnac interferometer as described in Refs. [30,36]. In this setup the strength of the measurement is controlled by rotating half-wave plate $\operatorname{HWP}(1)$ to $\theta$ and $\operatorname{HWP}(2)$ to $(\pi / 4)-\theta$, that are placed, respectively, in the path of horizontally and vertically polarized beams after the polarization beam splitter (PBS) such that $\eta=\cos (4 \theta)$. To switch between the bases $B_{y}$ according to the input $y$, Bob rotates both his waveplates $\mathrm{HWP}(\mathrm{B} 1)$ and $\mathrm{HWP}(\mathrm{B} 2)$ to $22.5^{\circ}$ and $0^{\circ}$, respectively. The outcome of these measurements $b \in\{0,1\}$ is encoded in the output path of the interferometer such that $b=0(b=1)$ corresponds to the detection of the photon in the output path " 1 " $\equiv$ transmission (" 2 " $\equiv$ reflection). In a sequential scenario, we choose to consider only one output path at a time to simplify the setup and by adding an additional rotation to the HWP(B1) and HWP(B2), we can 
select the output we want to analyze at a given time. Using output 2, Bob will locally be able to learn the outcome of his measurement counterfactually when using perfect detectors. Also, when the fair sampling assumption is invoked, which is the case in this experiment, Bob can still infer his outcome of the measurement locally using average photon rates.

Finally, Charlie's projective measurement setup consists of HWP(C), PBS, a pair of fiber couplers (FC) and multimode fibers (MMF) that propagate the photons to a pair of APDs. He performs $C_{z} \in\left\{\sigma_{x}, \sigma_{z}\right\}$ on the received qubits according to his random input $z \in\{0,1\}$, by rotating $\operatorname{HWP}(\mathrm{C})$ to $22.5^{\circ}$ and $0^{\circ}$, respectively. The results of Charlie's marginal probabilities (for evaluating $W_{\mathrm{AC}}$ ) are obtained by averaging out the inputs and outputs of Bob.

Results.-To evaluate $\left(W_{\mathrm{AB}}, W_{\mathrm{AC}}\right)$ from the data, we require the marginal probabilities appearing in Eq (1). All parties setting are set using motorized rotation stages that are controlled by a computer program. To gather sufficient statistics we measure $60 \mathrm{sec}$ in each setting with a rate of $\sim 20 \mathrm{kHz}$ and collected at least 1.2 million events. Each measured value of $\left(W_{\mathrm{AB}}, W_{\mathrm{AC}}\right)$ together with the (black color) error bars (horizontal and vertical corresponding to $W_{\mathrm{AB}}$ and $W_{\mathrm{AC}}$, respectively) is shown in Fig. 3 and can be compared to the optimal quantum correlations (red color) and the optimal classical correlations (blue color, given by $\left.\left(W_{\mathrm{AB}}, W_{\mathrm{AC}}\right) \leq 3 / 4\right)$. Our obtained quantum correlations are nearly optimal for all considered values of $\eta$. Also, in the worst case, the classical limit is outperformed by at least 15 standard deviations. Moreover, the data reliably outperforms the optimal quantum correlations attainable when Bob uses stochastic projective measurements (green color) (see Ref. [39]). This certifies the advantage of unsharp measurements in sequential QRACs. Notably, the projective bound is not outperfromed for the two data

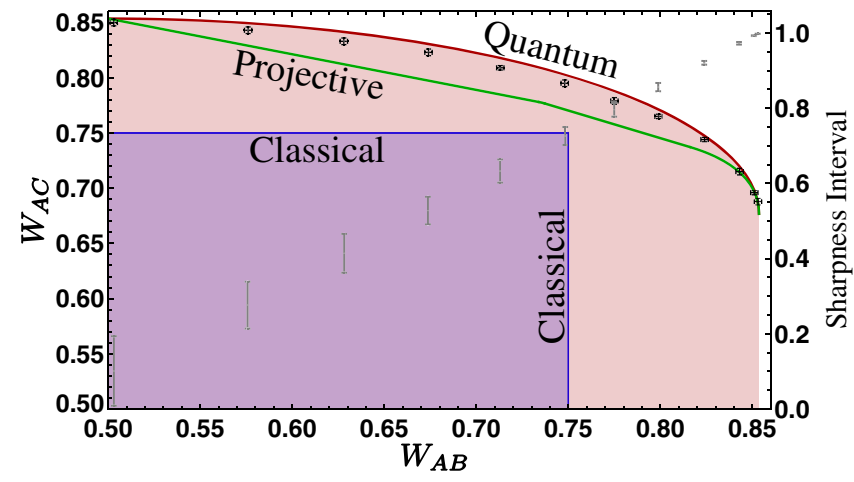

FIG. 3. Experimental results. Optimal quantum correlations (red), optimal quantum correlations from stochastic projective measurements (green), optimal classical correlations (blue), and experimentally obtained values of witness pairs $\left(W_{\mathrm{AB}}, W_{\mathrm{AC}}\right)$ (black). The characterization of the sharpness parameter $\eta$ is depicted by gray bars corresponding to the interval to which it is confined ( $y$ axis on the right-hand side). points corresponding to $\eta \in\{0,1\}$ since in these cases the bound coincides with the optimal quantum correlations.

From the inequalities in Eq. (3), we can determine an upper and a lower bound on the sharpness parameter. Thus, $\eta$ can be confined to the interval $I\left(W_{\mathrm{AB}}, W_{\mathrm{AC}}\right)$ for each of the measured values of the witness pair $\left(W_{\mathrm{AB}}, W_{\mathrm{AC}}\right)$. These certified intervals are depicted by gray bars in Fig. 3 located vertically from the corresponding witness' and using the $y$ axis on the right side. We observe that the certification is more precise (the interval is smaller) as the sharpness parameter increases. The smallest (largest) interval, corresponding to an essentially projective (noninteractive) measurement, has a width of about $10^{-3}(0.2)$. This is due to the fact that the bounds in Eq. (3) become more sensitive to small imperfections when $W_{\mathrm{AC}}$ increases. Further details about the experimental data is presented in the Supplemental Material [47]. Moreover, in Ref. [47], we also compare this characterization of unsharp measurements to a simple tomographic model with an essentially trusted preparation device subjected to comparably small errors.

Data analysis.-The experiment is influenced by systematic errors originating from, for instance, imperfect wave plates as well as offsets in their marked zero position, finite PBS extinction and cross talk, and limited interference visibility. The magnitude of these errors is revealed by the extent to which the experimental points are shifted away from the optimal quantum correlations. In order to minimize systematic errors, we carefully select and characterize all the optical components. This brings us closer to the optimal quantum correlation and the experimental points correspond to a more than $98 \%$ total visibility estimation. Nevertheless, random errors due to Poissonian statistics or due to repetition of the experimental settings with limited precision will spread the observed point on Fig. 3 to a region contained within the black bars. To keep this error low, all the settings are set by computerized controlled motors with repetition precision $<0.02^{\circ}$. Errors together with mean values are provided in the Supplemental Material [47].

Quantifying sequentual measurement incompatibility.In order to witness quantum correlations, one requires incompatible measurements. In that sense, violating the classical constraint with $W_{\mathrm{AB}}\left(W_{\mathrm{AC}}\right)$ certifies that Bob's (Charlie's) two POVMs are incompatible $[48,49]$. It is, however, more informative to consider a quantitative inference; is it possible to deduce from $\left(W_{\mathrm{AB}}, W_{\mathrm{AC}}\right)$ a lower bound on the extent to which Bob's and Charlie's POVMs are incompatible? In order to achieve such quantification of Heisenberg uncertainty, one must first define a measure of incompatibility valid for dichotomic qubit observables. We use the degree of incompatibility introduced in Ref. [44];

$$
D\left(\vec{n}_{0}, \vec{n}_{1}\right)=\left|\vec{n}_{0}+\vec{n}_{1}\right|+\left|\vec{n}_{0}-\vec{n}_{1}\right|-2,
$$


where $\vec{n}_{0}$ and $\vec{n}_{1}$ are the Bloch vectors of the observables. All compatible observables obey $D \leq 0$ whereas incompatible observables obey $D \leq 2(\sqrt{2}-1)$. As expected, the bound is saturated by two Pauli observables. Since we are interested in incompatible observables, we simply reset negative values of $D$ to 0 . As shown in Supplemental Material [47], the success rate of a QRAC implies a lower bound on $D$ :

$$
D \geq 8 W-6 .
$$

Thus, whenever a QRAC exceeds the classical bound of $\frac{3}{4}$, a degree of incompatibility is certified and quantified. By choosing $W=W_{\mathrm{AB}}$, we use Eq (6) to quantify the incompatibility of Bob's unsharp measurements. The bound in Eq. (6) can also be applied to Charlie's measurements, but it would significantly underestimate their degree of incompatibility due to the sequential nature of the experiment. A more sophisticated quantification is possible when exploiting both $W_{\mathrm{AB}}$ and $W_{\mathrm{AC}}$ and the fact that $\eta \in I\left(W_{\mathrm{AB}}, W_{\mathrm{AC}}\right)$. Considering unbiased observables for Bob, i.e., $B_{y}=\eta\left(\hat{n}_{y} \cdot \vec{\sigma}\right)$, where $\hat{n}_{y}$ is the normalized Bloch vector, we show in the Supplemental Material [47] that Charlie's degree of incompatibility respects

$$
D \geq \min _{\eta \in I\left(W_{\mathrm{AB}}, W_{\mathrm{AC}}\right)} \frac{16 W_{\mathrm{AC}}-8}{1+g_{\eta}+f_{W_{\mathrm{AB}}}\left(1-g_{\eta}\right)}-2
$$

where $g_{\eta} \equiv \sqrt{1-\eta^{2}}$ and $f_{W_{\mathrm{AB}}} \equiv 2\left(\eta_{\min } / \eta\right) \sqrt{1-\left(\eta_{\min } / \eta\right)^{2}}$.

Notice that if we choose not to exploit the certified interval $I\left(W_{\mathrm{AB}}, W_{\mathrm{AC}}\right)$, we may simply take the limit of $\eta \rightarrow 0$ and recover the bound in Eq. (6) for $W=W_{\mathrm{AC}}$. In Fig. 4 we show the degree of incompatibility as obtained from the twelve experimentally measured witness pairs $\left(W_{\mathrm{AB}}, W_{\mathrm{AC}}\right)$ corresponding to different targeted values of the sharpness parameter $\eta$. As expected, we see that the incompatibility of Bob's measurements decreases with $\eta$ and vanishes in the vicinity of $\eta=1 / \sqrt{2}$, which is the theoretical threshold. For Charlie, we always find a high

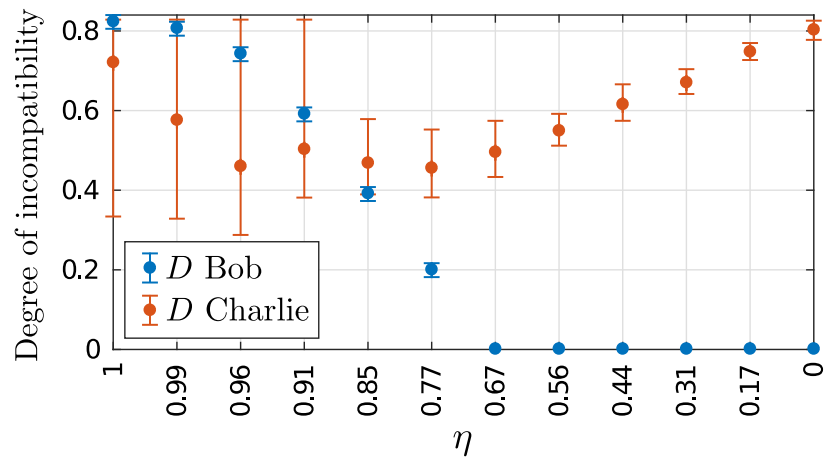

FIG. 4. Lower bound on the degree of incompatibility in Bob's (blue) and Charlie's (orange) respective pair of measurements for the twelve different targeted values of the sharpness parameter $\eta$. degree of incompatibility which stems from his projective measurements.

Conclusions.-By precise control of unsharp quantum measurements, we demonstrated nearly optimal sequential quantum random access codes that outperform not only the best possible classical protocols but also the best possible quantum protocols based only on projective measurements. We harvested the quantum advantage in the communication task in order to certify the degree of unsharpness in the preformed measurements. Exploiting both the sequential QRACs and the certification of the unsharpness, we quantitatively demonstrated the incompatibility of two sequential pairs of measurements across a wide range of sharpness parameters. Our results demonstrate the usefulness of unsharp measurements in quantum communication tasks, the possibility of quantifying the degree of incompatibility of sequential pairs of unsharp observables and the practical feasibility of characterizing them under weak assumptions.

This work was supported by the Swedish Research Council, Knut and Alice Wallenberg Foundation, and the Swiss National Science Foundation (Starting Grant DIAQ, NCCR-QSIT). N. M. acknowledges the financial support by the Foundation for Polish Science through the First Team Grant No. 2016-1/5.

[1] M. A. Nielsen and I. L. Chuang, Quantum Computation and Quantum Information, 10th anniversary ed. (Cambridge University Press, Cambridge, England, 2011).

[2] G. M. D'Ariano, P. L. Presti, and P. Perinotti, Classical randomness in quantum measurements, J. Phys. A 38, 5979 (2005).

[3] S. M. Barnett and S. Croke, Quantum state discrimination, Adv. Opt. Photonics 1, 238 (2009).

[4] R. Derka, V. Bužek, and A. K. Ekert, Universal Algorithm for Optimal Estimation of Quantum States from Finite Ensembles via Realizable Generalized Measurement, Phys. Rev. Lett. 80, 1571 (1998).

[5] J. M. Renes, R. Blume-Kohout, A. J. Scott, and C. M. Caves, Symmetric informationally complete quantum measurements, J. Math. Phys. (N.Y.) 45, 2171 (2004).

[6] J. B. Brask, A. Martin, W. Esposito, R. Houlmann, J. Bowles, H. Zbinden, and N. Brunner, Megahertz-Rate Semi-Device-Independent Quantum Random Number Generators Based on Unambiguous State Discrimination, Phys. Rev. Applied 7, 054018 (2017).

[7] E. S. Gómez et al., Device-Independent Certification of a Nonprojective Qubit Measurement, Phys. Rev. Lett. 117, 260401 (2016).

[8] A. Tavakoli, D. Rosset, and M-O. Renou, Enabling Computation of Correlation Bounds for Finite-Dimensional Quantum Systems via Symmetrisation, Phys. Rev. Lett. 122, 070501 (2019).

[9] J. M. Renes, Spherical-code key-distribution protocols for qubits, Phys. Rev. A 70, 052314 (2004). 
[10] M. Oszmaniec, L. Guerini, P. Wittek, and A. Acín, Simulating Positive-Operator-Valued Measures with Projective Measurements, Phys. Rev. Lett. 119, 190501 (2017).

[11] A. Tavakoli, M. Smania, T. Vértesi, N. Brunner, and M. Bourennane, Self-testing non-projective quantum measurements in prepare-and-measure experiments, Sci. Adv. 6, 16 (2020).

[12] R. Silva, N. Gisin, Y. Guryanova, and S. Popescu, Multiple Observers Can Share the Nonlocality of Half of an Entangled Pair by Using Optimal Weak Measurements, Phys. Rev. Lett. 114, 250401 (2015).

[13] A. N. Korotkov, Continuous quantum measurement of a double dot, Phys. Rev. B 60, 5737 (1999).

[14] J. Audretsch, T. Konrad, and A. Scherer, Sequence of unsharp measurements enabling a real-time visualization of a quantum oscillation, Phys. Rev. A 63, 052102 (2001).

[15] T. Konrad, A. Rothe, F. Petruccione, and L. Diósi, Monitoring the wave function by time continuous position measurement, New J. Phys. 12, 043038 (2010).

[16] K. W. Murch, S. J. Weber, C. Macklin, and I. Siddiqi, Observing single quantum trajectories of a superconducting quantum bit, Nature (London) 502, 211 (2013).

[17] A. A. Clerk, M. H. Devoret, S. M. Girvin, F. Marquardt, and R. J. Schoelkopf, Introduction to quantum noise, measurement, and amplification, Rev. Mod. Phys. 82, 1155 (2010).

[18] M. A. Armen, J. K. Au, J. K. Stockton, A. C. Doherty, and H. Mabuchi, Adaptive Homodyne Measurement of Optical Phase, Phys. Rev. Lett. 89, 133602 (2002).

[19] J. S. Lundeen, B. Sutherland, A. Patel, C. Stewart, and C. Bamber, Direct measurement of the quantum wavefunction, Nature (London) 474, 188 (2011).

[20] R. L. Cook, P. J. Martin, and J. M. Geremia, Optical coherent state discrimination using a closed-loop quantum measurement, Nature (London) 446, 774 (2007).

[21] G. G. Gillett, R. B. Dalton, B. P. Lanyon, M. P. Almeida, M. Barbieri, G. J. Pryde, J. L. O’Brien, K. J. Resch, S. D. Bartlett, and A. G. White, Experimental Feedback Control of Quantum Systems Using Weak Measurements, Phys. Rev. Lett. 104, 080503 (2010).

[22] C. Sayrin, I. Dotsenko, X. Zhou, B. Peaudecerf, T. Rybarczyk, S. Gleyzes, P. Rouchon, M. Mirrahimi, H. Amini, M. Brune, J-M. Raimond, and S. Haroche, Real-time quantum feedback prepares and stabilizes photon number states, Nature (London) 477, 73 (2011).

[23] O. Oreshkov and T. A. Brun, Weak Measurements Are Universal, Phys. Rev. Lett. 95, 110409 (2005).

[24] Y. Aharonov, D. Z. Albert, and L. Vaidman, How the Result of a Measurement of a Component of the Spin of a Spin-1/2 Particle can turn out to be 100, Phys. Rev. Lett. 60, 1351 (1988).

[25] Y. Ota, S. Ashhab, and F. Nori, Entanglement amplification via local weak measurements, J. Phys. A 45, 415303 (2012).

[26] F. J. Curchod, M. Johansson, R. Augusiak, M. J. Hoban, P. Wittek, and A. Acín, Unbounded randomness certification using sequences of measurements, Phys. Rev. A 95, 020102(R) (2017).

[27] A. Tavakoli and A. Cabello, Quantum predictions for an unmeasured system cannot be simulated with a finitememory classical system, Phys. Rev. A 97, 032131 (2018).
[28] A. Bera, S. Mal, A. Sen(De), and U. Sen, Witnessing bipartite entanglement sequentially by multiple observers, Phys. Rev. A 98, 062304 (2018).

[29] A. H. Shenoy, S. Designolle, F. Hirsch, R. Silva, N. Gisin, and N. Brunner, Unbounded sequence of observers exhibiting Einstein-Podolsky-Rosen steering, Phys. Rev. A 99, 022317 (2019).

[30] H. Anwer, N. Wilson, R. Silva, S. Muhammad, A. Tavakoli, and M. Bourennane, Noise-robust preparation contextuality shared between any number of observers via unsharp measurements, arXiv:1904.09766.

[31] A. Palacios-Laloy, F. Mallet, F. Nguyen, P. Bertet, D. Vion, D. Esteve, and A. N. Korotkov, Experimental violation of a Bell's inequality in time with weak measurement, Nat. Phys. 6, 442 (2010).

[32] F. Piacentini, A. Avella, M. P. Levi, M. Gramegna, G. Brida, I. P. Degiovanni, E. Cohen, R. Lussana, F. Villa, A. Tosi, F. Zappa, and M. Genovese, Measuring Incompatible Observables by Exploiting Sequential Weak Values, Phys. Rev. Lett. 117, 170402 (2016).

[33] Y. Kim, Y-S. Kim, S-Y. Lee, S-W. Han, S. Moon, Y-H. Kim, and Y-W. Cho, Direct quantum process tomography via measuring sequential weak values of incompatible observables, Nat. Commun. 9, 192 (2018).

[34] J.-S. Chen, M.-J. Hu, X-M. Hu, B.-H. Liu, Y.-F. Huang, C.-F. Li, C.-G. Guo, and Y.-S. Zhang, Experimental realization of sequential weak measurements of non-commuting Pauli observables, Opt. Express 27, 6089 (2019).

[35] M. Schiavon, L. Calderaro, M. Pittaluga, G. Vallone, and P. Villoresi, Three-observer Bell inequality violation on a twoqubit entangled state, Quantum Sci. Technol. 2, 015010 (2017).

[36] M.-J. Hu, Z.-Y. Zhou, X.-M. Hu, C.-F. Li, G.-C. Guo, and Y.-S. Zhang, Observation of non-locality sharing among three observers with one entangled pair via optimal weak measurement, Quantum Inf. 4, 63 (2018).

[37] G. Foletto, L. Calderaro, A. Tavakoli, M. Schiavon, F. Picciariello, A. Cabello, P. Villoresi, and G. Vallone, Experimental Certification of Sustained Entanglement and Nonlocality after Sequential Measurements, Phys. Rev. Applied 13, 044008 (2020).

[38] K. Mohan, A. Tavakoli, and N. Brunner, Sequential random access codes and self-testing of quantum measurement instruments, New J. Phys. 21, 083034 (2019).

[39] N. Miklin, J. J. Borkała, and M. Pawłowski, Semi-deviceindependent self-testing of unsharp measurements, Phys. Rev. Research 2, 033014 (2020).

[40] A. Ambainis, A. Nayak, A. Ta-Shama, and U. Varizani, Dense quantum coding and a lower bound for 1-way quantum automata, in Proceedings of 31 st ACM Symposium on Theory of Computing (Association for Computing Machinery (ACM), New York, 1999), pp. 376-383, https://doi.org/10.1145/301250.301347.

[41] A. Ambainis, D. Leung, L. Mancinska, and M. Ozols, Quantum random access codes with shared randomness, arXiv:0810.2937.

[42] A. Tavakoli, A. Hameedi, B. Marques, and M. Bourennane, Quantum Random Access Codes using Single d-Level Systems, Phys. Rev. Lett. 114, 170502 (2015). 
[43] A. Tavakoli, J. Kaniewski, A. Vértesi, D. Rosset, and N. Brunner, Self-testing quantum states and measurements in the prepare-and-measure scenario, Phys. Rev. A 98, 062307 (2018).

[44] P. Busch, P. Lahti, and R. F. Werner, Heisenberg uncertainty for qubit measurements, Phys. Rev. A 89, 012129 (2014).

[45] T. Heinosaari and M. Ziman, The Mathematical Language of Quantum Theory (Cambridge University Press, Cambridge, England, 2011).

[46] J-P. Pellonpää, Quantum instruments: I. Extreme instruments, J. Phys. A 46, 025302 (2013).
[47] See Supplemental Material at http://link.aps.org/supplemental/ 10.1103/PhysRevLett.125.080403 for experimental data, quantification of Bob's degree of incompatibility from a QRAC and quantification of Charlie's degree of incompatibility from two QRAC, and comparison to error-bounded detector tomography.

[48] A. Tavakoli and R. Uola, Measurement incompatibility and steering are necessary and sufficient for operational contextuality, Phys. Rev. Research 2, 013011 (2020).

[49] C. Carmeli, T. Heinosaari, and A. Toigo, Quantum random access codes and incompatibility of measurements, Europhys. Lett. 130, 50001 (2020). 